\title{
Rejuvenation of Ovary and Thin Endometrium by Autologous PRP Injection in POR and Recurrent Implantation Failure
}

\author{
Yazhini Selvaraj ${ }^{\circledR}$, Kanchana Malaisamy \\ Obstetrics and Gynecology, Ponni Hospital and Fertility Research Centre, Madurai, India \\ Email: yazhiniselvaraj1956@gmail.com,drkanchana100793@gmail.com
}

How to cite this paper: Yazhini, S. and Kanchana, M. (2021) Rejuvenation of Ovary and Thin Endometrium by Autologous PRP Injection in POR and Recurrent Implantation Failure. Advances in Sexual Medicine, 11, 1-15. https://doi.org/10.4236/asm.2021.111001

Received: December 15, 2020

Accepted: January 27, 2021

Published: January 30, 2021

Copyright $\odot 2021$ by author(s) and Scientific Research Publishing Inc. This work is licensed under the Creative Commons Attribution International License (CC BY 4.0).

http://creativecommons.org/licenses/by/4.0/

\section{(c) (i) Open Access}

\begin{abstract}
In India, the problem of infertility is growing and in the last 5 years, it has gone up to $20 \%-30 \%$. This ongoing prospective clinical study brings forth a novel, innovative, effective, simple, affordable, easily performed outpatient procedure $(\mathrm{OP})$ and a promising therapeutic method in rejuvenating the Ageing Ovaries and Thin Endometrium, with autologous Platelet Rich Plasma (PRP). This clinical study proves to give a better result in rejuvenating Ovary and treating the Thin Endometrium. This pilot study included five women (28 - 44 years) with Poor Ovarian Response (POR), Premature Ovarian Insufficiency (POI) and Perimenopause and Thirty-nine women (22 - 43 years.) with recurrent implantation failure due to Thin Endometrium were subjected to autologous PRP instillation under Ultrasound Guidance, and Hysteroscopic guided PRP. After PRP instillation into the ovaries a singinificant output was obtained with improved Anti Mullerian Hormone (AMH) and Antral Follicle Count (AFC) and out of five women three women concieved by Intra Cytoplasmic Sperm Injection (ICSI). PRP injected in women with Poor Ovarian Response found successful ovarian rejuvenation within 1 3 months and had a $60 \%$ of pregnancy rate, PRP into the endometrium imrproves the thickness and had resulted in $53.8 \%$ of successful pregnancies.
\end{abstract}

\section{Keywords}

Platelet Rich Plasma (PRP), Poor Ovarian Reserve (POR), Premature Ovarian Insufficiency (POI), Anti Mullerian Hormone (AMH), Antral Follicle Counts (AFC), Intra Cytoplasmic Sperm Injection (ICSI), Outpatient Procedure (OP)

\section{Introduction}

In India three decades ago, an inverted red triangle was found everywhere which symbolized family planning (small family norm), but now we could see a lot of 
fertility clinics and hospitals everywhere. What happened to our fertile population? Infertility is growing and in the last five years, it has gone up to $20 \%-30 \%$. Infertility has increased more in urban area than in rural, among Indian population. For every six-woman, one woman has infertility problem in Indian Urban areas. Nowadays Indian women when compared with Caucasians are aging faster almost five years ahead [1]. The fertility rate in a country is determined by calculating the average number of children that a woman will have during her reproductive years. Table 1 below shows the fertility rate and population growth rate from 1950 to 2020 in India. From 1880 to 1970, the fertility rate was consistent, which started to decrease from 1980 till date. In 2020 the fertility rate is decreased to 2.2 which is half the fertility rate of 1980 . The fertility rate has been declined to $0.9 \%$ when compared with 2019 fertility rate $(0.89 \%)$ according to the United Nations world population prospects. The Rejuvenation medicines help in promoting tissue regeneration and help to cure infertility [2].

Causes and How to Reduce Infertility? There are many causes for infertility. Some of the identified causes among women are postponing marriage and child birth, life style modification such as lack of exercise and sedentary life style, Obesity which leads to Poly Cystic Ovary Syndrome (PCOS), lack of sexual health awareness causing sexually transmitted diseases which affect the fertility [3], rampant use of emergency contraception and surgical abortions, serious infections which results in irreversible infertility, genital tuberculosis leading to tubal blocks and thin endometrium [4], diet containing chemicals and genetic mutants. The use of Environmental toxins such as plastics also results in infertility. Whereas on the other hand, rural women are also being affected because of being exposed to pesticides and fertilizers. When the technologies like mobile phones and laptops have made day to day life easier, it has also created negative impacts resulting in infertility. Smoking and consuming alcohol has now become

Table 1. Infertility rise in India from 1950-2020 based on the fertility rate and population growth rate.

\begin{tabular}{ccc}
\hline \multicolumn{3}{c}{ Infertility Rise in India } \\
\hline Year & Fertility Rate & Growth Rate \\
\hline 2020 & 2.200 & $-0.900 \%$ \\
2010 & 2.636 & $-2.950 \%$ \\
2000 & 3.346 & $-1.990 \%$ \\
1990 & 4.093 & $-2.060 \%$ \\
1980 & 4.857 & $-1.200 \%$ \\
1970 & 5.598 & $-1.100 \%$ \\
1960 & 5.894 & $-0.020 \%$ \\
1950 & 5.907 & $0.000 \%$ \\
\hline
\end{tabular}


common among men and women working in Information Technology (IT) which is not a common thing in India, and it is a cultural change that causes Infertility. $90 \%$ of male infertility remains unidentified. Following a proper diet (vegetables like green leafy vegetables, sprouts and beans and lentils) avoiding hormone injected chickens, maintaining a normal weight by exercising daily, avoiding alcohol, tobacco, stress and reducing the usage of caffeine reduces infertility to a large extent. Sexual education creates awareness among teens and young adults, which helps them to be cautious about sexually transmitted diseases and their impact on fertility, proper usage of mobile phones and laptops, help to reduce infertility at least to some extent. But still for some unknown causes of infertility which results in poor ovarian reserve and thin endometrium, a novel innovative method has become a rescue which is called Regenerative or Rejuvenation medicine, using Platelet Rich Plasma (PRP). The other Rejuvenation mechanisms are using Stem Cells and Adipocyte Derived Stem Cells (ASCS). Platelet Rich plasma (PRP) also known as autologous conditioned plasma, is a process in which the whole blood is centrifuged to obtain the concentrated platelets rich plasma protein removing the red blood cells [5]. PRP is composed of $94 \%$ of platelets and $5 \%$ of Red Blood Cells (RBC) remaining $1 \%$ of White Blood Cells (WBC).Several research states that Platelets with growth factors when injected into the ovaries have improved the oocyte production [6] [7]. PRP is an ongoing prospective clinical study that aims at improving Ovarian Rejuvenation and Thin Endometrium which causes recurrent implantation failure and investigates the autologous PRP's efficacy on POI animals [8] [9]. Igboeli, et al. found that autologous bone marrow mesenchymal stem cells transplanted into ovary of women rejuvenates the aging ovaries and resulted in production of healthy oocytes leading to successful pregnancies [10]. The use of PRP can be extended to treat male factor infertility such as Erectile Dysfunction, Oligo AsthenoTeratoSpermia (OATS), and to increase the Penial Growth (size). This clinical study was conducted between June 2018 to till date at Ponni Hospital and Fertility Research Centre in order to properly investigate efficacy of Autologous PRP into the Ovary in wide range of pathophysiological condition jeopardizing ovarian dynamic and also providing a pilot data regarding PRP application for Ovarian Rejuvenation and Thin Endometrium. In this study, five women of low AMH in the age group of 28 - 44 years were included, and Thirty-nine women of age 22 - 43 years were included who had repeated implantation failure. All received autologous PRP instillation under Ultra-sound guidance and about six received PRP through hysteroscopy for Thin Endometrium.

\section{Reproductive Medicine}

\subsection{About PRP}

PRP is the amount of plasma that contains platelet counts above baseline. PRP was first described by Kinsley in 1954, while conducting blood coagulation research initially, used in healing skin wound in rats. PRP in 1987 was used in an 
open-heart surgery in Italy, and also used in Dental and Plastic surgery in 1990, in mid-1990 it was used in Intra uterine, Sports Medicine, Cosmetic and Orthopedic Surgeries. In 2015 it was used in ovarian rejuvenation and Thickening of Thin Endometrium. Patients suffering with ovarian insufficiency are managed with In Vitro Fertilization (IVF) treatment, Oocyte Donation Program, Surrogacy or alternatively Adoption [11]. PRP plays a vital role in medical field such as Dermatology for anti-aging, Osteoarthritis, Chronic Tendinitis, Oral Surgery, Diabetic Foot and Ulcers, Musculo Skeletal Injuries, Augment Bone Graft Surgical wounds and Reproductive Medicine [12] [13]. Marx, et al. used PRP for treating patients who were affected with severe thrombopenia. Today PRP is used clinically in regenerative medicine in the field of antiaging therapy. PRP is a combination of many proteins like cytokines, hormones and growth factors, that serves as chemo attractants for stem cells, macrophages, neutrophils. These neutrophils are said to reach the wound site first to discard the debris and microbes in the wounded tissues [14] and results in post translational modification of $\approx 1500$ bio active factors.PRP Instillation into the endometrial cavity increases the endometrial growth and helps to overcome the problems of Thin Endometrium (Chang, et al.) [15]. PRP is cost-effective and can be offered to majority of infertile population in India. Emergence of Autologous platelet rich plasma (PRP) intraovarian infusion reflects a break-through approach, show casing promising results. The human ovary has an epithelial monolayer that sustains cyclic injury and local tissue repair during every ovulation, which is made possible by the resident stem cells that are thought to be crucial for the regeneration needed for hemostasis and organ integrity [16]. Some research has suggested that platelets may contribute to overall organ function as well. This Ovarian Rejuvenation not just solve fertility problems, it also diminishes some adverse effects. Ovarian rejuvenation procedure might create new oocyte in the ovaries of women who are not able to conceive because of low oocyte reserve, premature ovarian failure, or perimenopause. PRP is injected into the ovary to induce Ovarian Rejuvenation; the PRP application in the ovaries can stimulate the germ cell line developing into ovarian oocytes. The PRP injection in women with poor ovarian reserve found successful ovarian rejuvenation within 1 - 3 months. Similarly, the endometrium plays an important role in achieving optimal outcomes. Endometrial growth with PRP is a novel and promising method of improving pregnancy rate [17]. PRP is a safe enough OP procedure because of its autologous nature and also it is derived from the patient's own blood [18]. PRP has achieved the best of best result among the regenerative medicine. PRP has improved the clinical pregnancies by rectifying the thin endometrium and also helped in rejuvenating the aging ovaries along with Embryo Transfer (ET), which increased the live birth rates.

\subsection{Growth Factors in PRP}

Platelets activation process through PRP secretes multiple growth factors. These 
factors are essentially stored in thrombocyte a-granules and they have the key role in regulating cellular process, including chemotaxis, mitogenesis and differentiation [19]. These secreted growth factors directly stimulate local mesenchymal and epithelial cells to migrate, divide and increase the synthesis of collagen and matrix with resulting formation of fibrous connective tissue and scar formation. The growth factors when subjected to the impaired tissues, combines mixed actions to form an intact tissue. Platelet derived growth factor (PDGF) improves the collagen synthesis, proliferation of bone cells, fibroblast chemotaxis, Proliferative activity and Macrophage activation. The Vascular Endothelial Growth Factor (VEGF) triggers angiogenesis, migration, mitosis of endothelial cells, increases permeability of the vessels and stimulates chemotaxis of macrophages and neutrophils. The Transforming Growth Factor (TGF- $\beta$ ) enrich the synthesis of type I collagen, promotes angiogenesis, stimulates chemotaxis of immune cells, and inhibits osteoclast formation and bone resorption. The basic Fibro Blast growth factor (BFGF) advances the proliferation of mesenchymal cells, chondrocytes and osteoblasts. The Epidermal growth factor (EGF) enhances the cellular proliferation, differentiation of epithelial cells, promotes cytokine secretion by mesenchymal and epithelial cells. Insulin Like growth factor (IGF1 \& IGF2) stimulates cell growth, differentiation, recruitment in bone, blood vessel, skin and other tissues and stimulates collagen synthesis together with Platelet Derived Growth Factor (PDGF) [20] [21] [22] [23].

\section{Materials and Methods}

PRP is a simple OP procedure, which is done using PRP kit consisting of syringes, $14 \mathrm{ml}$ sterile conical bottom test tubes, sterile anticoagulant solution, sterile pipettes and centrifuge apparatus. Figure 1 illustrates the PRP preparation for both PRP instillation into ovary and endometrium. For PRP into endometrium take $14 \mathrm{ml}$ of conical bottom test tube in which collect $8.5 \mathrm{ml}$ of blood and $1.5 \mathrm{ml}$ of anticoagulant mix well and centrifuge for $15 \mathrm{~min}$ at $1200 \mathrm{rpm}$. For PRP instillation into ovary take $5 \mathrm{ml}$ of anticoagulant and $45 \mathrm{ml}$ of blood in an appropriate sterile test tube mix well and centrifuge for $15 \mathrm{~min}$ at $1500 \mathrm{rpm}$. There will be three layers after centrifugation, the bottom RBC's the middle platelets and WBC's the top serum. The suspended PRP will then be collected in another tube without mixing RBC's. Again, centrifuge at $3000 \mathrm{rpm}$ for 10 minutes to get the platelet pellet. Leave behind $1 \mathrm{ml}$ or $5 \mathrm{ml}$ of plasma above pellet and discard the remaining and use immediately. This procedure yields 7 - 10 times concentration of platelets in the final preparation, compared to blood in yielding growth factors. The second centrifugation is common for both endometrium and Ovaries.

\subsection{Selection Criteria}

Table 2 below gives the socio-demographic and clinical characteristics study of forty four women with Poor Ovarian Reserve and Thin Endometrium. This socio 


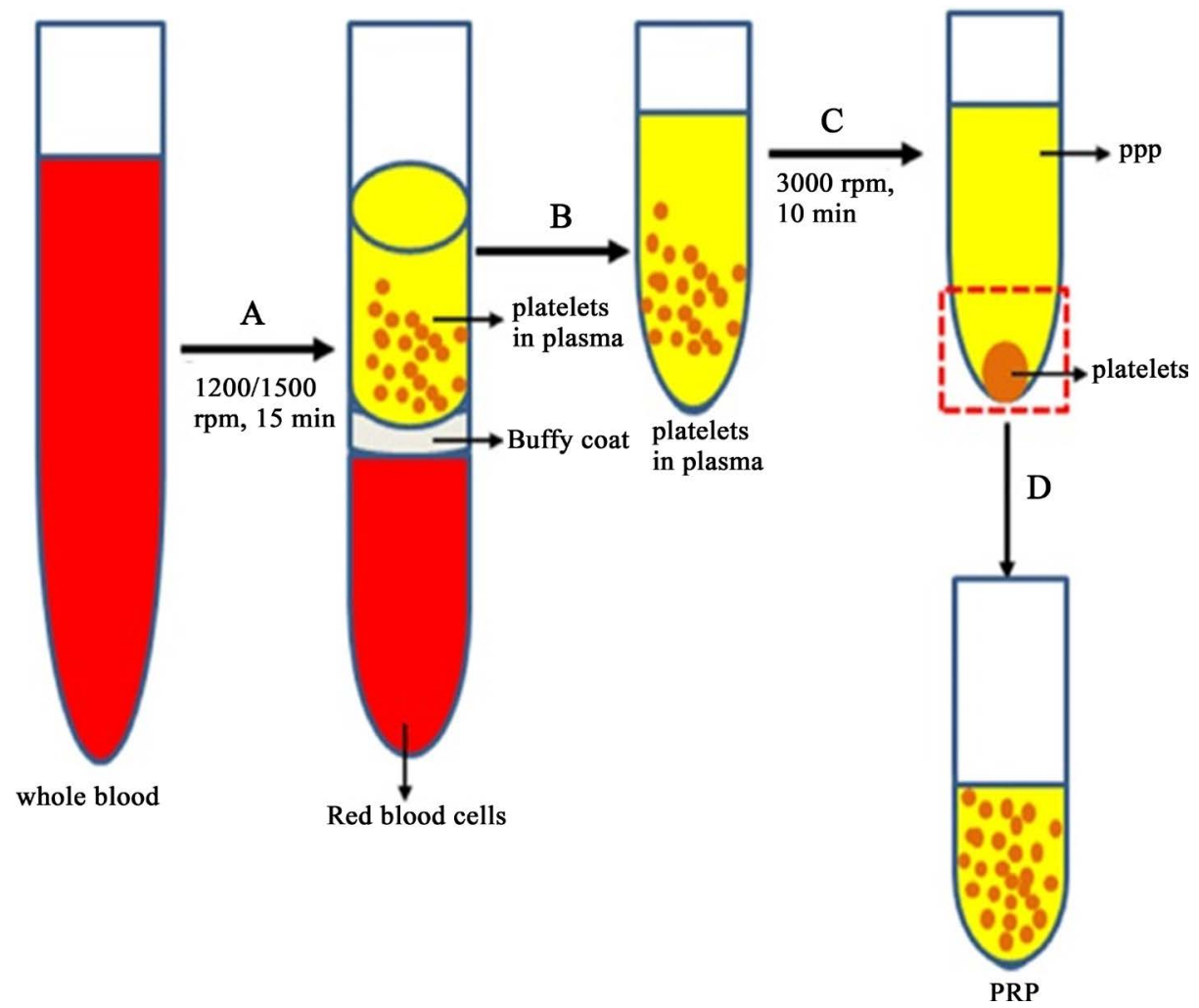

Figure 1. PRP preparation.

Table 2. Sociodemographic and clinical characteristics of patients with poor ovary reserve and thin endometrium.

\begin{tabular}{cc}
\hline & Sociodemographic \\
\hline Variables & $\mathbf{N}=\mathbf{4 4}$ \\
\hline Age & 12 \\
$\geq 40$ & 32 \\
$<40$ & \\
Education & 30 \\
Degree & 14 \\
$\leq 12$ th & \\
Religion & 39 \\
Hindu & 5 \\
Other Religion & \\
Married since & \\
$>10$ yrs & 18 \\
$\leq 10$ yrs & 26 \\
Ethnicity & \\
Indian & 22 \\
Non-Residential Indian &
\end{tabular}




\section{Continued}

Employment

$\begin{array}{lr}\text { Working } & 11 \\ \text { Homemaker } & 33 \\ \text { Income } & \\ \leq \text { Middle Class } & 33\end{array}$

Upper Middle Class

Clinical Characteristics

Infertility Type

Primary

Secondary

Luteinizing Hormone (LH)

$\begin{array}{ll}<5 & 10 \\ >5 & 34\end{array}$

Follicle Stimulating Hormone (FSH)

$\begin{array}{ll}\leq 5 & 13 \\ >5 & 21\end{array}$

A. Sociodemographic and clinical characteristics of the patients with Poor Ovarian Reserve and Thin Endometrium where $\mathrm{N}$ is the total no of patients.

demographic study includes educated women of above and below 40yrs, employed and unemployed, and women married since 10 yrs or below. The clinical characteristics of those women were done based on their type of infertility and level of Luteinizing hormone (LH) and Follicle Stimulating Hormone (FSH).

Ovary

The selection criteria for Poor Ovarian Responders include:

- Patient whose AMH level was very low which was less than 0.5 to $1.2 \mathrm{ng} / \mathrm{ml}$;

- Patients with a history of poor ovarian response having less than three eggs per cycle even after good stimulation with Gonadotrophins and patients with low estradiol levels;

- Antral Follicle Count of less than five from both ovaries;

- Patients with high FSH and LH levels does not have normal functioning ovaries, and who were in the menopausal stage;

- Lacking any other under lying disease causing female infertility;

- One patient had only one ovary and the other ovary had been removed due to torsion (Gangrenous).

\section{Endometrium}

The Selection criteria for Thin Endometrium includes:

- Cases with recurrent implantation failure due to thin endometrium;

- Patient who had at least one previously cancelled IVF due to thin endometrium or many attempts of IUI without success; 
- Patients with less than $6 \mathrm{~mm}$ endometrium, even after high dosage of estradiol, excluding Polyps, Fibroids and severe Asherman's syndrome;

- Like one of our patients whose age was 41 years, gave a history of six IUI and four IVF failure, was selected and was treated with PRP and she conceived and delivered a beautiful baby.

\subsection{Investigations}

The following preliminary investigations were done before PRP are:

- Urine culture and sensitivity;

- Vaginal swab for culture \& sensitivity;

- Preliminary Scan for AFC;

- Serum AMH;

- Hormones like FSH, LH, Estradiol, Progesterone, Prolactin to be tested;

- Thyroid Profile.

\subsection{Consent}

Informed and written consent was obtained from the couples' and their relatives.

\section{Why Intraovarian PRP?}

In the year 2017, 28 years old woman came from Malaysia landed in our hospital with severe pain abdomen and shock, she was married for three years. USG revealed right ovarian Torsion, Emergency Laparoscopy was done it revealed the right ovary to be gangrenous. So right ovariectomy done. She went back to Malaysia and in 2018 she came again but for infertility treatment giving history of five IUI, and one IVF failure. Her AMH was very low $0.5 \mathrm{ng} / \mathrm{ml}$ with Poor Antral Reserve in Left Ovary. She was treated with Dehydroepiandrosterone (DHEA), Lycopene, L-Arginine, Vitamin E, Vitamin D3, Vitamin C, Zinc, Melatonin, for three months with no improvement. We searched for a remedy and came to know about PRP through net. We learnt the technique of preparing PRP from Dr.Suriyakhant, Delhi who also introduced the kit needed for PRP. We under Aseptic Precautions did Intra Ovarian Instillation of PRP through Ultra Sound Guidance using ovum pickup needle without anesthesia. We waited for six weeks, and stimulated the patient with Recombinant FSH ( $\mathrm{rFSH}$ ), retrieved three eggs (M2), did ICSI and ET. She conceived and delivered a beautiful girl baby, which encouraged us to do Intra Ovarian PRP for Four more cases.

\section{Procedure}

PRP into the Ovaries was done with ultra sound guidance under Aspetic Precaution using $17 \mathrm{~g}$ ovum pick up needle. Inject $1-2 \mathrm{ml}$ of PRP into ovaries. For thin endometrium $0.5 \mathrm{ml}$ of PRP under Ultra Sound Guidance and aseptic precaution was instilled into the endometrium between day 7 and 11 and/or a day prior to Embryo Transfer (ET), twice or thrice using IUI cannula. In case of Hysteroscopic instillation through 10 to 12 punctures PRP was injected into the endometrium. 


\section{Results}

Table 3 below shows a comparison of patient's Luteinizing Hormone, Anti Mullerian Hormone, Follicle Stimulating Hormone and Estradiol levels before and after PRP. Among these five women with low AMH level, after receiving Intra Ovarian Instillation of PRP three women conceived by ICSI and one patient's $\mathrm{AMH}$ and AFC improved a lot but the patient didn't turn up for follow up due to transfer to another place and one failed whose age was 44 yrs. Table 4 and Table 5 show the patients endometrium thickness before and after PRP in IUI and IVF using ultra sound guidance and hysteroscopy. Among these thirty nine patients who had recurrent implantation failure due to thin endometrium twenty one conceived, eighteen live birth rates and three had abortions. PRP instillation into ovary and thin endometrium had a promising result of lowering the LH and FSH levels and increasing the thickness of endometrium to $7 \mathrm{~mm}$ and above, thereby rejuvenating the ovaries and thin endometrium. The conception rate in Ovarian $\mathrm{PRP}$ is $60 \%$ and that in endometrial PRP is $53.8 \%$.

Table 3. Intra ovarian PRP.

\begin{tabular}{cccccccccccc}
\hline \multicolumn{1}{c}{ Before PRP } & \multicolumn{5}{c}{ After PRP } \\
\hline Case & Age & Platelet & LH & AMH & FSH & E2 & LH & AMH & FSH & E2 & M2 \\
\hline 1 & 28 & $2 \mathrm{ml}$ & 12 & 0.5 & 10.6 & 98.4 & 3 & 1.3 & 6.1 & 610 & 3 \\
2 & 40 & $4 \mathrm{ml}$ & 14 & 0.50 & 12.4 & 46 & 2.5 & 2.6 & 5.5 & 398 & 2 \\
3 & 44 & $4 \mathrm{ml}$ & 15.1 & 1.2 & 5.11 & 70.4 & 2.2 & 0.2 & 3.2 & 277 & 3 \\
4 & 41 & $4 \mathrm{ml}$ & 18 & 0.49 & 16.5 & $<5.0$ & 3.2 & 1.7 & 4.7 & 376 & 2 \\
5 & 42 & $6 \mathrm{ml}$ & 22 & 0.08 & 25.2 & $<5$ & 2.9 & 2.4 & 7.5 & 332 & $2(\mathrm{M} 1)$ \\
\hline
\end{tabular}

A. Comparison of Luteinizing Hormone (LH), Anti-Mullerian Hormone (AMH), Follicular Stimulating Hormone (FSH) and Estradiol (E2) before and after PRP into Ovary.

\subsection{PRP into Endometrium in IUI}

Table 4. Endometrium thickness before and after PRP in IUI.

\begin{tabular}{cccccccc}
\hline \multicolumn{7}{c}{ Endometrium Thickness } \\
\hline $\begin{array}{c}\text { Total no } \\
\text { of cases }\end{array}$ & Age Group & $\begin{array}{c}\text { PRP } \\
\text { Method }\end{array}$ & $\begin{array}{c}\text { No. of } \\
\text { times }\end{array}$ & $\begin{array}{c}\text { Before } \\
\text { PRP }\end{array}$ & After PRP & $\begin{array}{c}\text { Result } \\
\text { pregnancy }\end{array}$ & PR\% \\
\hline & $25-30(14)$ & USG & Single 15 & 4.5 to 5.3 & 7.7 to $8.9 \mathrm{~mm}$ & $5+$ ve & $35.70 \%$ \\
& $31-35(5)$ & & Double 6 & 3.2 to 4.8 & 8.3 to $9.0 \mathrm{~mm}$ & $1+$ ve & $20 \%$ \\
21 & $36-40(2)$ & & Thrice 0 & 3.0 to 4.8 & 7.2 to $8.8 \mathrm{~mm}$ & $1+$ ve & $50 \%$ \\
& $41+($ nil) & & & Nil & nil & 0
\end{tabular}

SUCCESS RATE: $33.3 \%$

A. Endometrium Thickness before and after PRP in IUI.

\subsection{PRP into Endometrium in IVF}


Table 5. Endometrium thickness before and after PRP in IVF.

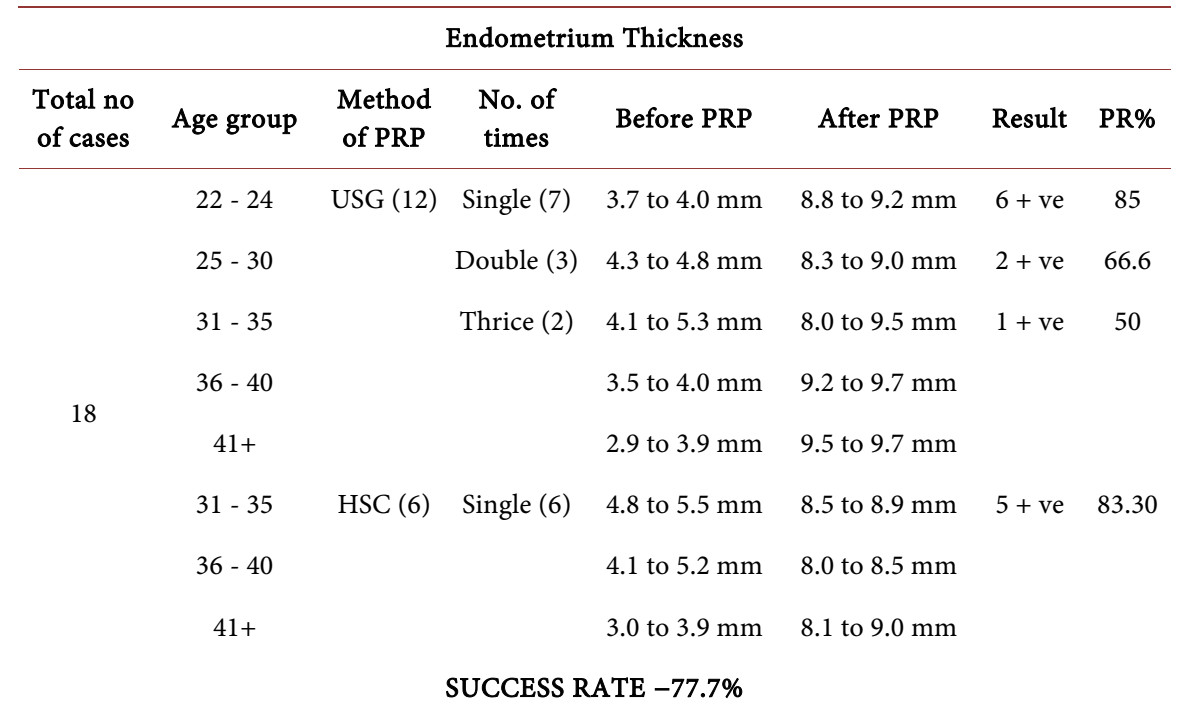

A. Endometrium Thickness before and after PRP in IVF using Ultra Sound Guidance (USG) and Hysteroscopy (HSC).

\section{Discussion}

Today PRP is clinically applied a lot in Regenerative Medicines The important aspect in assessing ovarian aging is with AFC and AMH [24] [25]. A comparative study is made with the results obtained from other researchers with PRP into Intraovarian and Thin Endometrium. Table 6 shows our results show much higher pregnancy rate, increase in AMH level and AFC after PRP when compared with some of the studies. First Data on In Vitro Fertilization and Blastocyst formation after Intra Ovarian injection of calcium gluconate activated autologous platelet rich plasma SillsEs, et al., USA, here a study was made among 4 women ( \pm 42 years.). Those women were suffering with Poor Follicular Response, Diminished Ovarian Reserve and Menopause, after $5 \mathrm{ml}$ of Autologous PRP into each ovary each patient had at least one blastocyst suitable for embryo preservation. The second comparison study was reactivating ovarian function through Autologous PRP Chin Med. 2020 Sfakianoudis K et al., Athens which was conducted with 120 women in total and thirty women under each category of POI, Poor Ovarian Reserve, Menopause and Perimenopausal woman. His study resulted with increased AMH levels and reduction in the FSH values and establishment of menstruation in menopausal women was achieved. Addressing Ovarian Insufficiency and the number of patients who responded were eighteen POI, thirteen menopause, twenty-four perimenopausal women. He reported one successful natural conception after PRP. The Third comparison study was made with Live births in women with Poor Ovarian Response through PRP, Marize Farimani, et al., Iran in which twenty three women with poor ovarian response were involved, nineteen women responded for $2 \mathrm{ml}$ of PRP infusion (two patients experienced spontaneous conception and one patient achieved through ART). 
Table 6. Comparison study for PRP instillaion in ovary.

\begin{tabular}{|c|c|c|c|c|c|c|}
\hline S.no & Study \& Author & No. of Patients & $\begin{array}{l}\text { No. of Patients } \\
\text { Responded }\end{array}$ & Category & PRP & Result \\
\hline 1 & $\begin{array}{l}\text { Sills Es, } \\
\text { et al., USA }\end{array}$ & 4 & 4 & $\begin{array}{c}\text { Menopause } \\
\text { POR } \\
\text { Diminished Ovarian Reserve }\end{array}$ & $\begin{array}{c}5 \mathrm{ml} \text { of Autologous } \\
\text { PRP }\end{array}$ & $\begin{array}{l}\text { Each patient had at least } \\
\text { one blastocyst suitable for } \\
\text { embryo preservation }\end{array}$ \\
\hline 2 & $\begin{array}{l}\text { Chin Med. } 2020 \\
\text { et al., Athens }\end{array}$ & 120 & $\begin{array}{c}\text { 18POI } \\
13 \text { Menopause } \\
\text { 24 Peri Menopause }\end{array}$ & $\begin{array}{c}\text { POI } \\
\text { Perimenopausal } \\
\text { Menopausal } \\
\text { Poor Responder Women }\end{array}$ & PRP Infusion & $\begin{array}{l}\text { Increased AMH, and } \\
\text { Reduction of FSH level, } \\
\text { achieved menstruation in } \\
\text { menopausal Woman }\end{array}$ \\
\hline 3 & $\begin{array}{l}\text { Marize Farimani } \\
\text { et al., Iran }\end{array}$ & 23 & 19 & POR & $2 \mathrm{ml}$ of PRP & $\begin{array}{l}2 \text { Spontaneous } \\
\text { Conception } 1 \text { clinical } \\
\text { pregnancy }\end{array}$ \\
\hline 4 & Pantos, K. et al. & 3 & 3 & $\begin{array}{c}\text { POR } \\
\text { Menopause } \\
\text { Perimenopause } \\
\text { Failed IVF }\end{array}$ & Autologous PRP & $\begin{array}{l}\text { Achieved pregnancy } \\
\text { through natural } \\
\text { conception within 2-6 } \\
\text { months\& improvement } \\
\text { in hormonal profile, FSH } \\
\text { level and AMH level. }\end{array}$ \\
\hline
\end{tabular}

The fourth comparison study was with Natural Conception resulting in ongoing pregnancies in menopausal and premature menopausal women with PRP treatment Pantos, K. et al., three women ( $\leq 46$ years.) with premature ovarian failure and menopausal woman with previously failed IVF were chosen. They were subjected to Autologous Ovarian PRP; all three patients achieved pregnancy through natural conceptions within 2 - 6 months and showed improvement in hormonal profile, FSH level, and AMH level. Finally, in our research we had five patients with POI and POR, who were treated with PRP instillation into ovary as needed and four patients showed improvement in AFC and AMH. Three patients conceived after ICSI, one patient failed whose age was $>40$ and one patient who showed good improvement didn't turn for follow up because of transfer to another place.

Likewise, a comparison study for clinical pregnancy rate after PRP in Thin Endometrium, is shown in below Table 7. The first comparative study for PRP in Endometrium was done with Chang, et al. in which five women with Thin Endometrium were treated with PRP infusion with Frozen Embryo Transfer and achieved hundred percentage success rates. The second study was did with Zadhemodarres et al., 2017, in which ten women were included for PRP treatment along with Frozen Embryo Transfer and resulted in fifty percentage success. The third study was made with Nazari, et al., 2016, in which ninety-seven women were chosen and forty-nine were subjected to PRP treatment and forty-eight were in control study. PRP infusion was done followed with frozen embryo transfer, resulted in $44.89 \%$ in the PRP group and $16.66 \%$ in the control group.

The fourth study was with Tandulwadkar, et al., 2017, where 68 women were done PRP with Embryo Transfer and achieved a clinical pregnancy rate of $45.3 \%$. (thirteen women with second trimester and thirteen women with first 
Table 7. Comparison Study for PRP instillaion in Endometrium.

\begin{tabular}{cccccccc}
\hline Study & $\begin{array}{c}\text { Type of } \\
\text { study }\end{array}$ & Partaker & Age & $\begin{array}{c}\text { Day of } \\
\text { PRP }\end{array}$ & $\begin{array}{c}\text { Average EMT } \\
\text { before PRP }\end{array}$ & $\begin{array}{c}\text { Average EMT } \\
\text { after PRP }\end{array}$ & $\begin{array}{c}\text { Clinical } \\
\text { PR Rate }\end{array}$ \\
\hline Chang et al., 2015 & Pilot & 5 & $35 \pm 4.0$ & 10 & $6.22 \mathrm{~mm}$ & $7.52 \mathrm{~mm}$ & $5 / 5$ \\
Zadhemodarres et al., 2017 & Pilot & 10 & $35 \pm 5.0$ & $11-12$ & $5.82 \mathrm{~mm}$ & $7.25 \mathrm{~mm}$ & $5 / 10$ \\
$\quad$ Nazari et al., 2016 & Pilot & 20 & $36 \pm 3.0$ & $16-18$ & N/A & N/A & $18 / 20$ \\
Tandulwadkar et al., 2017 & Pilot & 64 & $31 \pm 9.0$ & $15-16$ & $5.0 \mathrm{~mm}$ & $7.22 \mathrm{~mm}$ & $39 / 64$ \\
$\begin{array}{c}\text { Hounyoung et al., 2019 } \\
\text { Dr. Yazhini Selvaraj et al., }\end{array}$ & Pilot & 20 & $37.5 \pm 7.5$ & $12,15,18$ & $5.4 \pm 0.8 \mathrm{~mm}$ & $6.0+1.1 \mathrm{~mm}$ & $6 / 20$ \\
2020 & Pilot & 39 & $25-40$ & $7-14$ & $4.0 \mathrm{~mm}$ & $7-9 \mathrm{~mm}$ & $21 / 39$ \\
\hline
\end{tabular}

trimester, one ectopic pregnancy, three blastocysts, two had Blighted Ova, and two bio chemical pregnancies). The fifth study was made with Hounyoung, et al., 2019, in which 20 women were instilled PRP along with ET and $12.7 \%$ of implantation rate, clinical pregnancy rate $30 \%$ and live birth rate was $20 \%$. Our research study was done with 39 women out of which 21 were done PRP for IUI and 18 were done PRP for FET. Among 18, 6 were subjected to Hysteroscopic guided PRP and 12 were done with ultrasound guidance. The pregnancy rate when done under hysteroscopic guidance was $83.30 \%$ and ultra sound guidance was $75 \%$, overall, for FET the pregnancy rate was $77.7 \%$. All PRPs for IUI were done under ultra-sounded guidance either once or twice between day 7 to 11 , for single PRP the pregnancy rate was $35.70 \%$ and that of the double PRP it is $20 \%$, and the overall success of pregnancy rate is $33.3 \%$ which is much higher than the normal IUI pregnancy rate $18 \%-22 \%$. When all the studies with PRP was done prior to Embryo Transfer, ours is a unique research done not only for FET but also for IUI (21 cases).

\section{Conclusion}

Endometrium plays a vital role in achieving optimal outcome in ART. Current therapies offer little for patients with Thin Endometrium causing recurrent implant failure. PRP into Endometrial cavity improves the endometrial receptivity by increasing the progesterone receptor activity resulting in healthy endometrium and successful pregnancy (Chang, et al.). Endometrial growth with PRP is a novel innovative effective and promising therapeutic method to improve its thickness and thereby the pregnancy rate. In a total of 44 patients of both Intraovarian PRP and PRP into Endometrium, 23 patients conceived. PRP injected in woman with poor ovarian reserve paved the way to successful ovarian rejuvenation within 1 - 3 months had resulted in $60 \%$ of successful pregnancy rate and PRP into the endometrium had $53.8 \%$ successful pregnancies. All patients who conceived were followed up to delivery. We have not encountered any complications. It is a simple, affordable and easily performed OP procedure which gives encouraging results. It is a boon to the couple who earnestly desire their own genetic component offspring and brings hope to women suffering from Poor 
Ovarian Reserve, POF, Perimenopause and related issues like thin endometrium leading to recurrent implantation failure. PRP the newer field of rejuvenation medicine brings the Ray of Hope, for many unsolved problems in infertile couple.

\section{Conflicts of Interest}

The authors declare no conflicts of interest regarding the publication of this paper.

\section{References}

[1] Sills, E.S., Rickers, N.S., Li, X. and Palermo, G.D. (2018) First Data on in Vitro Fertilization and Blastocyst Formation after Intraovarian Injection of Calcium Gluconate-Activated Autologous Platelet Rich Plasma. Gynecological Endocrinology, 34, 756-760. https://doi.org/10.1080/09513590.2018.1445219

[2] Farimani, M., Heshmati, S., Poorolajal, J. and Bahmanzadeh, M. (2019) A Report on Three Live Births in Women with Poor Ovarian Response Following Intra-Ovarian Injection of Platelet-Rich Plasma (PRP). Molecular Biology Reports, 46, 1611-1616. https://doi.org/10.1007/s11033-019-04609-w

[3] Olooto, W.E., Amballi, A.A. and Banjo, T.A. (2012) A Review of Female Infertility: Important Etiological Factors and Management. Journal of Microbiology and Biotechnology Research, 2, 379-385.

[4] Bos-Mikich, A., de Oliveira, R. and Frantz, N. (2018) Platelet-Rich Plasma Therapy and Reproductive Medicine. Journal of Assisted Reproduction and Genetics, 35, 753-756. https://doi.org/10.1007/s10815-018-1159-8

[5] Zadehmodarres, S., Salehpour, S., Saharkhiz, N. and Nazari, L. (2017) Treatment of Thin Endometrium with Autologous Platelet-Rich Plasma: A Pilot Study. JBRA Assisted Reproduction, 21, 54. https://doi.org/10.5935/1518-0557.20170013

[6] Pantos, K., Simopoulou, M., Pantou, A., Rapani, A., Tsioulou, P., Nitsos, N., Syrkos, S., Pappas, A., Koutsilieris, M. and Sfakianoudis, K. (2019) A Case Series on Natural Conceptions Resulting in Ongoing Pregnancies in Menopausal and Prematurely Menopausal Women Following Platelet-Rich Plasma Treatment. Cell Transplantation, 28, 1333-1340. https://doi.org/10.1177/0963689719859539

[7] Abdullah, T.H., Abbas, S.H., Al-Obaidi, M.T. and Abdulraheem, Y. (2019) The Efficacy of Platelets Rich Plasma (PRP) for Ovarian Rejuvenation. Indian Journal of Public Health Research \& Development, 10, 1211-1217. https://doi.org/10.5958/0976-5506.2019.02060.6

[8] Molina, A., Sánchez, J., Sánchez, W. and Vielma, V. (2018) Platelet-Rich Plasma as an Adjuvant in the Endometrial Preparation of Patients with Refractory Endometrium. JBRA Assisted Reproduction, 22, 42. https://doi.org/10.5935/1518-0557.20180009

[9] Ahmadian, S., Sheshpari, S., Pazhang, M., Bedate, A.M., Beheshti, R., Abbasi, M.M., Nouri, M., Rahbarghazi, R. and Mahdipour, M. (2020) Intra-Ovarian Injection of Platelet-Rich Plasma into Ovarian Tissue Promoted Rejuvenation in the Rat Model of Premature Ovarian Insufficiency and Restored Ovulation Rate via Angiogenesis Modulation. Reproductive Biology and Endocrinology, 18, 1-13. https://doi.org/10.1186/s12958-020-00638-4

[10] Prosper, I., El Andaloussi, A., Sheikh,U., Takala, H., ElSharoud, A., McHugh, A., Gavrilova-Jordan, L., Levy, S. and Al-Hendy, A. (2020) Intraovarian Injection of autologous Human Mesenchymal Stem Cells Increases Estrogen Production and Re- 
duces Menopausal Symptoms in Women with Premature Ovarian Failure: Two Case Reports and a Review of the Literature. Journal of Medical Case Reports, 14, $1-11$.

[11] Pantos, K., Nitsos, N., Kokkali, G., Vaxevanoglou, T., Markomichali, C., Pantou, A., Grammatis, M., Lazaros, L. and Sfakianoudis, K. (2016) Ovarian Rejuvenation and Folliculogenesis Reactivation in Peri-Menopausal Women after Autologous Platelet-Rich Plasma Treatment. Abstracts, ESHRE32nd Annual Meeting, Athens, 3-6.

[12] Willemsen, J.C.N., van der Lei, B., Vermeulen, K.M. and Stevens, H.P.J.D. (2014) The Effects of Platelet-Rich Plasma on Recovery Time and Aesthetic Outcome in Facial Rejuvenation: Preliminary Retrospective Observations. Aesthetic Plastic Surgery, 38, 1057-1063. https://doi.org/10.1007/s00266-014-0361-Z

[13] Fernandez-Moure, J.S., Van Eps, J.L., Cabrera, F.J., Barbosa, Z., del Rosal, G.M. Weiner, B.K., Ellsworth IV, W.A. and Tasciotti, E. (2017) Platelet-Rich Plasma: A Biomimetic Approach to Enhancement of Surgical Wound Healing. Journal of Surgical Research, 207, 33-44. https://doi.org/10.1016/j.jss.2016.08.063

[14] Pavlovic, V., Ciric, M., Jovanovic, V. and Stojanovic, P. (2016) Platelet Rich Plasma: A Short Overview of Certain Bioactive Components. Open Medicine, 11, 242-247. https://doi.org/10.1515/med-2016-0048

[15] Chang, Y.J., Li, J.J., Chen, Y.Q., Wei, L.N., Yang, X., Shi, Y.N. and Liang, X.Y. (2015) Autologous Platelet-Rich Plasma Promotes Endometrial Growth and Improves Pregnancy Outcome during in Vitro Fertilization. International Journal of Clinical and Experimental Medicine, 8, 1286.

[16] Sfakianoudis, K., Simopoulou, M., Grigoriadis, S., Pantou, A., Tsioulou, P., Maziotis, E., Rapani, A., et al. (2020) Reactivating Ovarian Function through Autologous Platelet-Rich Plasma Intraovarian Infusion: Pilot Data on Premature Ovarian Insufficiency, Perimenopausal, Menopausal, and Poor Responder Women. Journal of Clinical Medicine, 9, 1809. https://doi.org/10.3390/jcm9061809

[17] Nazari, L., Salehpour, S., Hosseini, M.S. and Moghanjoughi, P.H. (2019) The Effects of Autologous Platelet-Rich Plasma in Repeated Implantation Failure: A Randomized Controlled Trial. Human Fertility, 23, 209-213. https://doi.org/10.1080/14647273.2019.1569268

[18] Farimani, M., Bahmanzadeh, M. and Poorolaja, J. (2016) A New Approach Using Autologous Platelet-Rich Plasma to Treat Infertility and to Improve Population Replacement Rate. Journal of Research in Health Sciences, 16, 172.

[19] Kim, H., Shin, J.E., Koo, H.S., Kwon, H., Choi, D.H. and Kim, J.H. (2019) Effect of Autologous Platelet-Rich Plasma Treatment on Refractory Thin Endometrium during the Frozen Embryo Transfer Cycle: A Pilot Study. Frontiers in Endocrinology, 10, 61. https://doi.org/10.3389/fendo.2019.00061

[20] Agarwal, M., Mettler, L., Jain, S., Meshram, S., Günther, V. and Alkatout, I. (2020) Management of a Thin Endometrium by Hysteroscopic Instillation of Platelet-Rich Plasma into the Endomyometrial Junction: A Pilot Study. Journal of Clinical Medicine, 9, 2795. https://doi.org/10.3390/jcm9092795

[21] Kobayashi, E., Flückiger, L., Fujioka-Kobayashi, M., Sawada, K., Sculean, A., Schaller, B. and Miron, R.J. (2016) Comparative Release of Growth Factors from PRP, PRF, and Advanced-PRF. Clinical Oral Investigations, 20, 2353-2360.

https://doi.org/10.1007/s00784-016-1719-1

[22] Tandulwadkar, S.R., Naralkar, M.V., Surana, A.D., Selvakarthick, M. and Kharat, A.H. (2017) Autologous Intrauterine Platelet-Rich Plasma Instillation for Suboptimal Endometrium in Frozen Embryo Transfer Cycles: A Pilot Study. Journal of 
Human Reproductive Sciences, 10, 208. https://doi.org/10.4103/jhrs.JHRS 2817

[23] Xie, X.T., Zhang, C.Q. and Tuan, R.S. (2014) Biology of Platelet-Rich Plasma and Its Clinical Application in Cartilage Repair. Arthritis Research \& Therapy, 16, 204. https://doi.org/10.1186/ar4493

[24] Liu, C.H., Jiang, H., Zhang, W.X. and Yin, H.Q. (2017) Double Ovarian Stimulation during the Follicular and Luteal Phase in Women $\geq 38$ Years: A Retrospective Case-Control Study. Reproductive BioMedicine Online, 35, 678-684.

https://doi.org/10.1016/j.rbmo.2017.08.019

[25] Şükür, Y.E., Kıvançli, İ.B. and Özmen, B. (2014) Ovarian Aging and Premature Ovarian Failure. Journal of the Turkish German Gynecological Association, 15, 190. https://doi.org/10.5152/jtgga.2014.0022 